\title{
Restauración atraumática para el control de la caries dental: historia, características y aportes de la técnica
}

\author{
Jorge Tascón $n^{1}$
}

Forma de citar

Tascón J. Restauración atraumática para el control de la caries dental: historia, características y aportes de la técnica. Rev Panam Salud Publica. 2005;17(2):110-5.

RESUMEN Este trabajo presenta información de relevancia científica sobre la historia, características y aportes de la técnica de restauración atraumática en la prevención y el control de la caries dental.

Dentro del ámbito de la salud pública bucodental, la técnica de restauración atraumática ha sido desde hace muchos años una medida económica y eficaz para la prevención y el control de la caries en poblaciones vulnerables. Permite, entre otras cosas, reducir el estrés y la ansiedad que generan los métodos convencionales de restauración. Vistas las limitaciones de su aplicación en cavidades dentales de dos o más superficies, se recomienda promover más la investigación sobre esta técnica, que promete grandes beneficios en los países latinoamericanos, con el propósito de mejorar su eficacia a partir de sus propias características, indicaciones y fundamentos técnicos.

Palabras clave Odontología en salud pública, odontología comunitaria, odontología preventiva, caries dental, cementos de ionómero vítreo, restauración dentaria provisoria.

La caries dental es la enfermedad bacteriana que históricamente ha afectado con mayor constancia a la cavidad bucal en las poblaciones humanas $\mathrm{y}$, aunque su prevalencia ha disminuido (1), sigue siendo el mayor problema de salud bucodental en la

\footnotetext{
Departamento de Investigaciones y Salud Pública, Colegio Odontológico Colombiano, Cali, Colombia. Escuela de Salud Pública, Facultad de Salud, Universidad del Valle, Cali, Colombia. Toda correspondencia deberá dirigirse a: Jorge Tascón, Colegio Odontológico Colombiano, Calle 13 Norte No. 3 N 13, Santiago de Cali (Valle), Colombia. Tel: (572) 6612410; fax: (57-2) 6613051. Dirección electrónica: jtascon@odontologico.edu.co
}

mayor parte de los países industrializados. Por lo demás, afecta a una proporción de incluso 60 a $90 \%$ de la población escolar y adulta de todo el mundo (2-8). En la actualidad, la distribución y gravedad de la caries varía de una región a otra y su frecuencia muestra una firme relación con factores socioculturales, económicos, del ambiente y del comportamiento (9).

En Colombia, la caries es la enfermedad bucal de mayor prevalencia y sus efectos se intensifican en proporción con la edad $(10,11)$. Según datos del III Estudio Nacional de Salud Bucal realizado en este país, el índice
COP-D (suma de dientes [D] con caries [C]; dientes con obturación [O] permanente y dientes perdidos $[\mathrm{P}]$ por caries, dividida entre el total de individuos examinados) alcanza valores de 2,3 en niños de 12 años y de 5,2 en adolescentes de 15 a 19 años (10).

Si a los datos anteriores se añaden los efectos psicosociales de la caries (12) y su impacto en términos de morbilidad, pérdida de dientes y costo de los tratamientos de tipo operatorio y de rehabilitación bucal (13), además de la frecuente dificultad de acceso a los servicios odontológicos (14), resulta evidente la necesidad de adoptar téc- 
nicas clínicas que puedan llegar a toda la población, a fin de lograr una mejor prevención de este importante trastorno bucal.

En palabras del Director General de Sanidad de Estados Unidos (15), la desatención de los problemas bucodentales se traduce en dolor y sufrimiento innecesarios, complicaciones devastadoras para el bienestar general de la persona y costos económicos y sociales que rebajan considerablemente la calidad de vida de las sociedades.

En su informe sobre salud bucal de 1997 (16), la Organización Panamericana de la Salud recalcó que la salud bucal es un aspecto fundamental de las condiciones generales de salud en las Américas, debido al peso específico que añade a la carga global de morbilidad, a los altos costos de su tratamiento y a la posibilidad, aun no aprovechada plenamente, de realizar intervenciones eficaces para la prevención de la caries dental.

A lo largo de la historia se han probado diversas intervenciones para el tratamiento de la caries, con el propósito natural de disminuir la pérdida de piezas dentarias. En tiempos recientes, en Noruega, por ejemplo, durante los años 40 y 50 la caries rampante se eliminaba de los dientes deciduos realizando abrasión de la estructura dental con discos de carburo (especialmente en las zonas interproximales), después de lo cual las lesiones se trataban con nitrato de plata.

Con la finalidad también de reducir la incidencia de caries, en los años 50 fue práctica usual en Escandinavia realizar exodoncia temprana de los primeros molares permanentes, a fin de crear en la dentición permanente, diastemas que permitieran una mejor higiene bucal (17).

Entre los diversos recursos empleados a través de la historia para el control de la caries destaca la técnica de restauración atraumática (TRA), que a mediados de los años 80 fue probada en África y en el decenio de 1990 se incorporó de manera definitiva, con el aval de la Organización Mundial de la Salud y con el apoyo del gobierno holandés, en los programas de salud dental de Tailandia, China y países de África (17).

\section{ORIGEN Y CLASIFICACIÓN DE LA TÉCNICA}

La técnica de restauración atraumática se desarrolló dentro del marco del programa de atención primaria de la salud bucal de la Escuela Dental de Dar es Salaam, en la República de Tanzania, África, en respuesta a la necesidad de encontrar un método para preservar los dientes cariados en personas de todas las edades que viven en países en desarrollo y en comunidades menos favorecidas. La duración lograda se ha estimado en cinco años, según los estudios publicados (18). Intervenciones similares se emprendieron luego en Tailandia (sureste asiático) en 1991, Zimbabwe (África) en 1993 y Pakistán (sur de Asia Central) en 1995 y en China en el año 2001 (19); de igual manera, se han llevado a cabo proyectos de intervención en los que se ha aplicado la técnica de restauración atraumática en países latinoamericanos como Perú y Ecuador.

En la técnica de restauración atraumática, los tejidos cariados se retiran con instrumentos manuales y luego la cavidad resultante, así como las fosetas y fisuras adyacentes, se restauran y sellan con un material adhesivo, por lo general ionómero de vidrio (20-22).

En el proceso de sellado, a manera de extensión de la técnica de restauración atraumática, los dientes con fosetas y fisuras profundas, así como aquellos con caries incipientes (en el esmalte), se sellan con ionómero de vidrio de alta densidad $(20,21)$.

A diferencia de los métodos convencionales, la técnica de restauración atraumática es indolora en la mayor parte de los casos, no requiere el uso de equipo odontológico eléctrico y ofrece eficacia a bajo costo (23).

\section{EXPERIENCIA EN LA APLICACIÓN DE LA TÉCNICA}

La técnica de restauración atraumática se ha empleado particularmente en comunidades de bajos recursos, grupos especiales con incapacidad mental o física, escuelas, centros de salud con carencias de equipo odonto-
CUADRO 1. Algunas ventajas y desventajas de la técnica de restauración atraumática para el control de la caries dental

Ventajas

- Se elimina solamente el tejido reblandecido infectado (esmalte y dentina)

- Requiere mínima preparación de la cavidad, según lo determina la forma de la lesión

- Evita la necesidad de anestesia local, porque es una técnica indolora

- Simplifica el control de infecciones cruzadas, porque es fácil lavar y esterilizar los instrumentos

- No requiere equipos eléctricos ni hidráulicos, de alto costo

- Permite sellar fosetas y fisuras

Desventaja principal

- Ofrece poca eficacia en la restauración de cavidades de más de dos superficies

Fuente: Datos de las referencias 23 y 24.

lógico y poblaciones asentadas en regiones remotas (24). El empleo creciente de esta técnica de restauración ha permitido conocer con mayor claridad sus ventajas, indicaciones y contraindicaciones (cuadros 1 y 2).

\section{MATERIAL EMPLEADO EN LA TÉCNICA: IONÓMERO DE VIDRIO}

\section{Generalidades}

El ionómero de vidrio, parte fundamental de la técnica de restauración atraumática, fue sintetizado por los in-

\section{CUADRO 2. Principales indicaciones y con- traindicaciones de la técnica de restaura- ción atraumática}

Indicaciones

- Lesiones cariosas leves y moderadas

- Lesiones cariosas accesibles con instrumentos manuales

Contraindicaciones

- Presencia de absceso dental. No debe utilizarse en estos casos

- Pulpa dental expuesta. No debe emplearse en estos casos

Fuente: Datos de las referencias 20 a 24. 
gleses A.D. Wilson y B.E. Kent en 1969 (25). De acuerdo con Sueo Saito, citado por Carrillo (2000), la palabra ionómero (del griego ion, 'átomo o partícula con carga' y meros, 'miembro de una clase específica') designa un polímero que forma enlaces covalentes dentro de las cadenas largas, y enlaces iónicos entre ellas. Son esas características las que confieren a este material su gran poder de adhesión.

Para el uso clínico, la preparación del cemento de ionómero de vidrio consiste en la mezcla de dos componentes, uno en polvo y otro líquido. El polvo es un vidrio especial, compuesto básicamente de flúor, aluminio y silicio, que debe sus propiedades opalescentes a la presencia de fluoruro de calcio. El líquido, que es una solución electrolítica de copolímeros con radicales carboxilo, recibe el nombre químico de ácido polialquenoico (26).

La reacción del vidrio con el ácido polialquenoico produce el desplazamiento de iones positivos de $\mathrm{Ca}^{+} \mathrm{Al}^{+}$ (cationes eléctricamente positivos) y de iones con carga negativa, como el fluoruro (25). De estas polisales del vidrio, la de calcio se forma primeramente, como un gel de consistencia firme, que puede tallarse. Luego, la formación de policarboxilato de aluminio confiere a este material restaurativo una consistencia dura, de roca $(25,26)$.

La reacción descrita, que se encuadra en el concepto químico de las reacciones acidobásicas, incluye la liberación de un subproducto, el ion fluoruro, al cual se debe el efecto anticariogénico que ofrece este tipo de cemento (27)

\section{Características clínicas del ionómero de vidrio}

Las principales ventajas y desventajas $(26,28,29)$ del ionómero de vidrio en su aplicación clínica se resumen en los cuadros 3 y 4.

\section{TÉCNICA CLÍNICA}

Se coloca al paciente en posición supina sobre una mesa, escritorio o
CUADRO 3. Principales ventajas del ionómero de vidrio

- Alta biocompatibilidad

- Buenas propiedades físicomecánicas

- Buena adherencia a sustratos dentarios (esmalte, dentina y cemento)

- Mínima contracción al polimerizar

- Propiedades aislantes, térmicas y eléctricas

- Buen sellado marginal

- Facilidad de aplicación

- Anticariogénico por liberación de flúor y por su actividad antimicrobiana

Fuente: Datos de las referencias 26,28 y 29.

CUADRO 4. Principales desventajas del ionómero de vidrio

- Difícil pulimento

- Resistencia subóptima al agua

- Alto riesgo de microfiltración marginal

y fractura en cavidades compuestas

- Limitaciones estéticas

Fuente: Datos de las referencias 26,28 y 29 .

cama. El operador elimina el tejido reblandecido por medio de excavación con instrumento manual. Una vez retirado el tejido cariado y aislado con algodón el campo operatorio, se aplica el ionómero de vidrio para obturar la cavidad y para sellar las fosetas y fisuras adyacentes. Ya que en esta técnica no se utilizan instrumentos rotatorios, es necesario ajustar el contorno del diente y la oclusión antes de que se complete la polimerización del material restaurativo (18). Cabe puntualizar aquí que el éxito de las restauraciones elaboradas con la técnica de restauración atraumática depende fundamentalmente de una buena capacitación y de la estandarización de criterios entre el personal que la aplique.

\section{APORTES DE LA TÉCNICA AL CONTROL Y LA PREVENCIÓN DE LA CARIES DENTAL}

Además de las ventajas técnicas que representan su rápida aplicación, la buena humectación y su adhesión química al tejido dental duro, el ionómero de vidrio, como todo material biocom- patible que libere flúor, favorece la remineralización de las estructuras dentarias obturadas y de las que se encuentran adyacentes $(30,31)$.

De igual manera, estudios in vitro han demostrado una menor colonización y adhesión microbiana en las zonas restauradas con este material, en comparación con otros como la amalgama y la resina compuesta. Estas características implican la inhibición del proceso carioso, en la medida en que impiden la formación y acumulación de placa bacteriana, con el consecuente debilitamiento del material restaurador (32).

La literatura recopila investigaciones $(21,33)$ que se han propuesto evaluar la durabilidad de los tratamientos basados en la técnica de restauración atraumática. Estudios que abarcan un tiempo breve (p. ej., un año) señalan cifras de buenos resultados de 80 a $95 \%$ en la restauración de cavidades de una sola superficie en las clases I y V, de 55 a $75 \%$ en la clase II, y de 32 a $55 \%$ en las clases III y IV.

En un metaanálisis que recopiló la información publicada hasta septiembre de 2003, Frencken, Amerongen y Holmgren (2004) dividieron la literatura en dos períodos (publicaciones de 1987 a 1992 y de 1995 en adelante). El análisis concluyó que en el primer período las restauraciones de una superficie realizadas con amalgama presentaron mayor durabilidad que las realizadas con la técnica de restauración atraumática, después de uno, dos y tres años de seguimiento. Sin embargo, en el segundo período evaluado no se encontraron diferencias estadísticamente significativas entre los dos métodos de restauración (34).

Holmgren y Lo (2001) señalaron que el éxito de la técnica, a dos años y medio de haberse aplicado en una población preescolar china, fue de $75 \%$ en cavidades con clasificación de Black (35) I y $\mathrm{V}$ y de $27,5 \%$ en aquellas con más de una superficie o compuestas (36). De igual manera, estudios realizados a dos años (18) en la población adulta señalaron que hasta $7 \%$ de las restauraciones habían fracasado principalmente por caries recurrente o por desgaste o fractura del ionómero de vi- 
drio, que fue el material empleado en las restauraciones. Al mismo tiempo, $34,5 \%$ de los sellos aplicados habían fracasado.

En estudios que abarcaron plazos de tres años $(23,37,38)$, la eficacia de las restauraciones de una superficie y la de las restauraciones compuestas y de más de una superficie se estimó en 82,5 y $48,7 \%$, respectivamente. Al comparar este método con la técnica convencional de restauración con amalgama, se encontraron diferencias estadísticamente significativas en eficacia: $82,5 \%$ con la técnica atraumática y $79,6 \%$ con la técnica convencional.

\section{Implicaciones psicológicas y fisiológicas}

En comparación con el adulto, la perspectiva de un tratamiento odontológico suscita en el niño un grado bastante mayor de inquietud y temor. De esa manera, para lograr la cooperación del paciente infantil el profesional de la salud bucal debe acudir a técnicas de control del comportamiento (39-42), ya sean psicológicas (como el modelado, decir-mostrar-hacer, el control por voz) o farmacológicas (como la sedación o la anestesia general). Luego, para mantener esa cooperación es fundamental que los procedimientos clínicos sean rápidos y confortables, lo cual se consigue con el uso de materiales de restauración que: a) requieran preparación mínima de la cavidad y b) sean fáciles de aplicar, al tiempo que c) muestren un grado aceptable de adherencia a la estructura dentaria, d) no se desprendan fácilmente y e) ofrezcan propiedades adecuadas de resistencia y desgaste (43).

En la técnica de restauración atraumática se utiliza el ionómero de vidrio reforzado porque este, además de cumplir con los requisitos señalados en el párrafo anterior, es un material restaurativo de autocurado (se endurece solo) que posee propiedades preventivas. Por ser precisamente atraumática para el paciente, esta técnica de restauración evita en gran medida la ansiedad que se asocia con los procedimientos clínicos invasores, los cuales requieren el uso de la fresa (de la pieza de mano) y de inyecciones (de anestésico), que son los principales factores determinantes del miedo en la población. ${ }^{2}$

Schuller y colaboradores (44) demostraron que los pacientes con puntuaciones altas de miedo por los tratamientos odontológicos (en la escala Corah Dental Anxiety) presentan un mayor número de dientes y superficies cariadas y el antecedente de un menor número de visitas al odontólogo, en comparación con aquellos que obtuvieron puntaciones bajas.

La técnica es atraumática tanto para el paciente (al procurar comodidad y bienestar durante el procedimiento) como para la pieza dentaria que se restaura, en la medida en que posibilita una amplia conservación del tejido sano adyacente a la caries y respeta el tejido pulpar. En una investigación de 359 pacientes de 6 a 16 años de edad, Amerongen y Rahimtoola (45) obtuvieron informes de menor incomodidad y dolor cuando la caries se eliminó con la técnica de restauración atraumática que cuando se retiró con la técnica convencional con instrumentos rotatorios. La profundidad de las preparaciones realizadas con instrumentos manuales fue comparativamente menor.

De igual manera, Schiriks y Amerongen (2003) evaluaron el grado de incomodidad, en términos del dolor, la ansiedad y el comportamiento registrados, entre 403 niños de seis y siete años de edad, durante procedimientos restaurativos realizados con instrumentos rotatorios y con la técnica de restauración atraumática. Durante todo el tratamiento los niños del grupo que se trató con la técnica atraumática tuvieron una puntuación más baja en la escala de Venham, lo que significa mayor comodidad y relajación durante el procedimiento. Aun así, resulta de interés que al comparar la frecuencia cardíaca entre los dos grupos no se advirtieron diferencias estadísticamente significativas (46).

\footnotetext{
(Tascón J. El Modelo de Creencias en Salud en el estudio de la caries en adolescentes del Valle del Cauca. [Trabajo de investigación]. Universidad del Valle, Colombia: 2003)
}

\section{CONCLUSIONES}

Por sus características clínicas y por el tipo de materiales empleados, la técnica de restauración atraumática resulta ser un método muy viable para la prevención y detención del proceso carioso fuera del ámbito de las clínicas tradicionales. En comparación con los métodos convencionales, la técnica de restauración atraumática encuentra mayor aceptación entre la población infantil y adolescente, por cuanto genera menos estrés y ansiedad, gracias a que evita el uso de la pieza de mano y a que la eliminación del tejido cariado no suele requerir anestesia. Dada la eficacia de esta técnica, parece muy recomendable su aplicación en poblaciones de escasos recursos que habitan en zonas con acceso limitado a los servicios de salud, y en personas o poblaciones que no están protegidas por los sistemas de seguridad social o de salud pública. No obstante, es importante precisar que en la medida en que estas personas o poblaciones cuenten con la protección de dichos sistemas, debe procurarse la realización de tratamientos definitivos convencionales, con amalgama, particularmente en el caso de cavidades compuestas (de más de una superficie), con el propósito de aumentar la durabilidad de las restauraciones.

El ionómero de vidrio reforzado, que es el material restaurativo y sellador de mayor uso en esta técnica, favorece también la prevención y el tratamiento del proceso carioso, por su capacidad de liberar flúor y por sus propiedades muy favorables de adhesión química al tejido dentario.

Entre las desventajas del ionómero de vidrio - que son las propias de los cementos de base acuosa- se encuentran su relativa aspereza y su resistencia subóptima al agua inmediatamente después de la colocación. Por otro lado, son importantes sus limitaciones estéticas y los riesgos de microfiltración marginal y fractura en cavidades compuestas, debido a que con el tiempo adquiere una textura similar a la de la tiza. Las desventajas anteriores impiden elegir el ionómero de vidrio como material restaurativo definitivo en cavidades compuestas. 
De acuerdo con lo publicado hasta la fecha, durante los primeros tres años las técnicas de obturación con amalgama y de restauración atraumática no muestran diferencia estadísticamente significativa en cuanto a la permanencia de las restauraciones en cavidades de una superficie de dientes permanentes. Sin embargo, por las limitaciones de durabilidad ya señaladas, la técnica de restauración atraumática no es apropiada para el tratamiento definitivo de cavidades de más de una superficie o compuestas.
Sin perder de vista esas consideraciones, el hecho de que la técnica de restauración atraumática se haya instaurado ya en varios países donde priman los criterios de una buena relación eficacia-costo, mínima intervención y máxima prevención, parece apoyar nuestra recomendación de que los planes para la investigación de la técnica se extiendan también a Colombia, como se ha hecho ya en otros países de características similares (Ecuador, Uruguay y Panamá). La intención de acrecentar los cono- cimientos sobre este método e, idealmente, identificar el material que le imparta una eficacia comparable a la de las técnicas convencionales, independientemente del tipo de cavidad o el número de superficies que se encuentren afectadas.

Agradecimento. El autor expresa su más sincero agradecimiento a Jo Frencken, de la Universidad de Nijmegen, Países Bajos, por sus valiosos aportes acerca de la técnica de restauración atraumática.

\section{REFERENCIAS}

1. Bönecker M, Cleaton-Jones P. Trends in dental caries in Latin American and Caribbean 5-6 and 11-13 year old children: A systematic review. Community Dent Oral Epidemiol. 2003;31:152-7.

2. Petersen P. The World Oral Health Report 2003: Continuous improvement of oral health in the 21st century-the approach of the WHO Global Oral Health Programme. Community Dent Oral Epidemiol. 2003;31(Suppl. 1):3-5.

3. Pinkham Jr. Odontología pediátrica. 2. ${ }^{\mathrm{a}}$ ed. Santa Fe de Bogotá: Interamericana; 1994.

4. Silverstone L. Caries dental: etiología, patología y prevención. México, D.F.: Manual Moderno; 1995.

5. Katz S. Odontología preventiva en acción. México, D.F.: Panamericana; 1975.

6. Gamboa L. Epidemiología de la caries. En: Universitas Odontol. 2000;20.

7. Jiménez L, Hernández M, Alemán N. Situación de la salud bucal de 20 familias pertenecientes al consultorio 30-2 [citado: $10 \mathrm{feb}$ 2002]. Hallado en: http://capiro.vcl.sld.cu/ medicentro/v3n299/sit.htm

8. Buenaño L, Salcedo O, Arizmendi B. Identificación de factores que influyen en la aparición de la caries de la lactancia. En: Rev Estomatol 1996;6:39-44.

9. World Health Organization. Global Oral Health Data Bank. Ginebra: WHO; 2002 [citado: 12 nov 2004]. Hallado en: http:// www.whocollab.od.mah.se/>

10. Colombia, Ministerio de Salud. III Estudio Nacional de Salud bucal-ENSAB III. Santa Fe de Bogotá: Ministerio de Salud; 1999.

11. World Health Organization. Oral Health Country/Area Profile Programme Department of Noncommunicable Disease Surveillance/ Oral Health WHO Collaborating Centre, Malmö University. Malmö (Suecia): WHO; 2004 [citado: 04 abr 2003]. Hallado en: http:// whocollab.od.mah.se/expl/orhdmft.html

12. Yip H, Smales R, Yu C, Gao X, Deng D. Comparison of atraumatic restorative treatment and conventional cavity preparations for glass ionomer restorations in primary molars: one year results. Quintessence Int. 2002;33: $17-21$.

13. Oscarson N, Källestal C, Fjekddahl A, Lindholm L. Cost effectiveness of different caries preventive measures in a high-risk population of Swedish adolescents. Community Dent Oral Epidemiol. 2003;31:169-78.

14. Brenes W, Sosa D. Epidemiología bucal y accesibilidad a los servicios odontológicos de un grupo de adolescentes. En: Rev Costarric Cienc Med. 1986;7:311-4.

15. U.S. Department of Health and Human Services. Oral health in America: a report of the Surgeon General-executive summary. Rockville, MD: DHHS, National Institute of Dental and Craniofacial Research. National Institutes of Health, 2000 [citado: 04 abr 2003]. Hallado en: http:/ /www2.nidcr.nih.gov/sgr/ execsumm.htm (En español: http://www. odontologiapreventiva.com/part Three 2000)

16. Organización Panamericana de la Salud. Salud oral. 16 julio 1997. CD40/20 (Esp.) [citado: 04 abr 2003] Hallado en: http:// www. paho.org/Spanish/GOV/CD/doc259.pdf

17. Mjör I, Gordan V. A review of atraumatic restorative treatment (ART). Int Dent J. 1999; 49:127-31.

18. Tak Ho T, Smales R, Taksang D. A two year clinical study of two glass ionomer cements used in the attraumatic restorative treatment (ART) technique. Community Dent Oral Epidemiol. 1999;27:195-201.

19. Lo ECM, Holmgren CJ. Provision of atraumatic restorative treatment (ART) restorations to Chinese pre-school children: a 30month evaluation. Int J Paed Dent. 2001; 11:3-10.

20. Frencken J, Holmegren C. How effective is ART in the management of dental caries? Community Dent Oral Epidemiol. 1999;27: 423-30.

21. Ewoldsen N, Cacho Z, Callagan S. Atraumatic restorative treatment using a mixture of glass ionomer cements. Rev Asoc Dent Mex. 1999; $56: 8-11$.
22. Massara M, Alves J, Brandao P. Atraumatic restorative treatment: clinical, ultrastructural and chemical analysis. Caries Res. 2002;36: 430-6.

23. Taifur D, Frenken J, Biruti N, Hof M, Truin G. Effectiveness of glass ionomer (ART) and amalgam restorations in the deciduous dentition: results after 3 years. Caries Res. 2002;36: $437-44$.

24. WHO Oral Health Country/Area Profile Programme. Introducing the atraumatic restorative treatment [citado: 04 abr 2003]. Hallado en: http://www.whocollab.od.mah.se/expl/ art2.html

25. Guzmán H. Biomateriales odontológicos de uso clínico. Santa Fe de Bogotá: Ecoe Ediciones; 1999.

26. Carrillo C. Actualización sobre las cementos de ionómero de vidrio, 30 años (1969-1999). Rev Asoc Dent Mex. 2000;57(2):65-71.

27. McLean J, Wilson AD. The development of glass ionomer cements: formulations and properties. Austral Dent J. 1977;22(1).31-6.

28. Ewoldsen N, Cacho Z, Callahan S, Froeschle $\mathrm{M}$, Brackett M. Tratamiento restaurador no traumático usando una mezcla de cementos de ionómero de vidrio. Rev Asoc Dent Mex. 1999;56:8-11.

29. Smales $R$, Yip $H$. The atraumatic restorative treatment (ART) approach for the management of dental caries. Quintessence Int. 2002; 33:427-32.

30. Bynum A, Donly K. Enamel de/remineralization on teeth adjacent to fluoride releasing materials without dentifrice exposure. J Dent Child. 1999;1:89-92.

31. Jang K, García-Godoy F, Donly K, Segura A. Remineralizing effects of glass ionomer restorations on adjacent interproximal caries. J Dent Child. 2001;1:125-8.

32. Kitsuyuki K, Susuki J, Okada M, Nagasaka N. In vitro study of antibacterial and antiadhesive activities of fluoride containing lightcured fissure sealants and a glass ionomer liner/base against oral bacteria. J Dent Child. 2000;1:117-22. 
33. Smales $\mathrm{R}$, Yip $\mathrm{H}$. The atraumatic restorative treatment (ART) approach for primary teeth: review of the literature. Pediatr Dent. 2000;22: 294-7.

34. Frencken J, Amerongen W, Holmgren CJ. Effectiveness of single surface ART restorations in the permanent dentition: a meta-analysis. J Dent Res. 2004;83:120-3.

35. Gilmore W. Odontología operatoria. México, D.F.: Interamericana; 1976.

36. Lo ECM, Holmgren CJ. Provisión of atraumatic restorative treatment (ART) restorations to Chinese pre-school children-a 30 month evaluation. Int J Paed Dent. 2001;11: 3-10.

37. Frencken J, Makoni F, Sithole W. ART restorations and glass ionomer sealants in Zimbabwe: survival after 3 years. Community Dent Oral Epidemiol. 1998;26:372-81.

38. Taifour D, Frencken JE, Beiruti N, Van't Hof MA, Truin GJ, Gelderman W, et al. Comparison between restorations in the permanent dentition produced by hand and rotary instrumentation-survival after 3 years. Community Dent Oral Epidemiol. 2003;31:122-8.

39. Cadavid P, Giaimo C. Eficacia de la técnica de modelado con vídeo preoperatorio en pacientes de 4 a 6 años de edad [citado: 30 oct 2004]. Hallado en: http://www.encolombia. com/focvol57no19699-odontopediatria4. $\mathrm{htm}>$

40. Cárdenas J. Pautas para el manejo no farmacológico del comportamiento en el paciente pediátrico [citado: 30 oct 2004] Hallado en: http:/ / www.encolombia.com/ortopedivol 197-guiademanejo9-2.htm>

41. López C. Guía para el manejo del paciente pediátrico bajo sedación interactuada [citado: 30 oct 2004] Hallado en: http://www. encolombia.com/ortopedivol197-guiade manejo9-3.htm>

42. Cadavid AL. Anestesia general: una estrategia de manejo en odontopediatría [citado: 30 oct 2004] Hallado en: http://www. encolombia.com/ortopedivol197-guiade manejo9-4.htm>
43. Thean H, Mok B, Chew C. Bond strenghts of glass ionomer restoratives to primary vs. permanent dentin. ASDC J Dent Child. 2000;67 (2):112-6.

44. Schuller A, Willumsen T, Holst D. Are there differences in oral health and oral health behavior between individuals with high and low dental fear? Community Dent Oral Epidemiol. 2003;31:116-21.

45. Amerongen $\mathrm{W}$, Rahimtoola S. Is ART really atraumatic? Community Dent Oral Epidemiol. 1999;27:431-5.

46. Schiriks M, Amerongen W. Atraumatic perspectives of ART: psychological and physiological aspects of treatment with and without rotary instruments. Community Dent Oral Epidemiol. 2003;31:15-20.

Manuscrito recibido el 12 de julio de 2004. Aceptado para publicación, tras revisión, el 30 de noviembre de 2005.

ABSTRACT This paper presents relevant scientific information on the history, characteristics, and contributions of atraumatic restorative treatment (ART) for use in preventing and controlling dental caries.

Within the area of oral public health, ART has been for years an economical, effec-

Atraumatic restorative tive method for preventing and controlling caries in vulnerable populations. Among treatment to control dental caries: history, characteristics, and contributions of the technique other things, ART reduces the stress and anxiety in patients that conventional restoration methods produce. This technique promises major benefits for Latin America. However, given its limitations with dental cavities on two or more surfaces, it is recommended that more research on this approach be encouraged, with the aim of improving the technique's effectiveness based on its characteristics, indications, and technical merits.

Keywords Public health dentistry; community dentistry; preventive dentistry; dental caries; glass ionomer cements; dental restoration, temporary.

Laughter is the most healthful exertion.

[Reír es la actividad más sana.] 